\title{
Educational
}

Forum

\section{L-Glutamic acid and glutamine: Exciting molecules of clinical interest}

\author{
C. Kulkarni, K. S. Kulkarni, B. R. Hamsa
}

Department of Pharmacology,

St. John's Medical College, Bangalore, India

\begin{abstract}
Glutamine is one of the most abundant amino acids and participates in a variety of physiological functions, namely - as a major fuel source for enterocytes, as a substrate for neoglucogenesis in kidney, lymphocytes, and monocytes, a nutrient/substrate in muscle protein metabolism in response to infection, inflammation, and muscle trauma. Studies evaluating the role of glutamine have confirmed it's participation in maintaining mucosal integrity of the gastrointestinal tract following it's administration in patients with major bowel surgery. The role of glutamine as a protective agent in hepatobiliary dysfunction and as a supplement in total parenteral nutrition is well established, particularly, in patients under intensive care.

L-Glutamic acid (L-GA) physiologically exists as glutamate. Glutamate along with glutamine plays a major role in amino acid metabolism and thus in maintaining nitrogen balance in the body. Glutamate is a well-established excitatory neurotransmitter in the central nervous system. There has been convincing evidence on protective activity of L-GA and $\alpha$ ketoglutarate in vincristine-induced neurotoxicity.

Based on the above information, a large number of studies have been carried out. The findings of recent clinical studies are presented below. Looking at the wide profile of activity, it has been proposed that though L-GA and glutamine were once considered nonessential for health, may now be considered as - 'conditionally essential' amino acids. While complete therapeutic role is yet to be elucidated, it may be anticipated that L-GA and glutamine may prove to be exciting molecules of interest to clinicians. The future research may therefore be directed at confirming the above activities and at investigating their role in other clinical conditions.
\end{abstract}

KEY WORDS: Amino acid; glutamic acid; glutamine.

\section{Introduction}

L-Glutamic acid (L-GA), a seaweed ingredient, identified in 1908 by Japanese scientists responsible for enhancing flavor for food is now best known scientifically as monosodium glutamate (MSG). The sodium salt of glutamic acid (GA) is called as MSG. ${ }^{[1]}$ The other names include - S-(+)-GA, L-GA, 2aminoglutaric acid, and an anionic form of MSG at physiological pH known as glutamate. ${ }^{[2]}$

MSG, a derivative of GA, is reported to be a naturally occurring nutrient in many foods and is increasingly used in food processing and home cooking in the western world. ${ }^{[3]}$ Its commercial use to improve food palatability for humans is well documented. ${ }^{[4]}$ The presence of free form of glutamate, not linked to protein is said to enhance flavor in food. ${ }^{[5]}$

MSG is known to produce a unique taste sensation termed 'UMAMI' the fifth taste, i.e. savory or brothy taste present in tomatoes and cheese. Free glutamate content is said to increase during the process of natural ripening and bring about a fuller taste in many foods, the basis behind is not known. ${ }^{[1]}$ It is also called as 'palate pleaser.' Studies on taste have shown that infants to adults enjoy taste of foods containing glutamate, which is said to be 10 times more in breast milk compared to cow's milk.

Glutamine is the most abundant amino acid present in the body. It is also known as levoglutamide, L-GA 5 amide, L-(+)2-aminoglutamicacid. ${ }^{[6]}$ It is synthesized in the body from GA and ammonia in an energy requiring reaction. Although nonessential in health, glutamine is conditionally essential in stress and illness. ${ }^{[2]}$

Studies over the last several years have explored the physiological role and therapeutic utility of these molecules in various disease conditions. In this review, an attempt is made 
to briefly discuss the results of these studies and highlight the future potential for therapeutic utility of L-GA and glutamine, which are physiologically and biochemically inter-related.

\section{Physicochemical properties}

GA is a dicarboxylic acid ${ }^{[2]}$ and is grouped under branched chain amino acid with acidic chain. ${ }^{[7]} \mathrm{L}-\mathrm{GA}$ is an aliphatic amino acid undergoing degradation in the body to glutamine, which is an amino acid with uncharged polar side chains.

\section{Glutamate}

$$
\begin{array}{ll}
\text { Molecular formula } & -\mathrm{C}_{5} \mathrm{H}_{9} \mathrm{NO}_{4} \\
\text { Appearance } & - \text { White crystalline powder } \\
\text { Melting point } & -205^{\circ} \mathrm{C} \\
\text { Stability } & - \text { Stable, incompatible with strong } \\
& \text { oxidizing agents }
\end{array}
$$

At physiological pH, GA exists in anionic form and is referred to as glutamate. It is found on surfaces of proteins and it plays a central role in transamination reactions and equilibrates with corresponding keto acid.

It is odorless and is best classified as food additive by Joint Expert Committee on food additives advisory board to WHO and FAO. ${ }^{[1]}$

\section{Glutamine}

Molecular formula - $\mathrm{C}_{5} \mathrm{H}_{10} \mathrm{~N}_{2} \mathrm{O}_{3}$

Appearance

- White crystalline powder/ white crystals

Stability

- Unstable ${ }^{[9]}$

\section{Synthesis and metabolism in the human body}

\section{Glutamate}

Human brain is the only tissue which has the highest content of glutamate. It is obtained from glucose via Krebs cycle intermediate. It is stored in synaptic vesicles, released by calcium-dependent exocytosis and is said to act as an endogenous agonist of the receptors mentioned in Table $1 .^{[10]}$

Glutamate is also synthesized in the body through transamination of $\alpha$-ketoglutarate by accepting amino groups from amino acids, first step in metabolism of amino acid. ${ }^{[7]}$

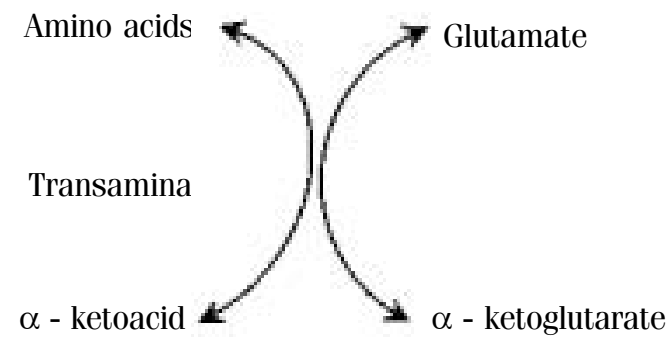

\section{Catabolism}

GA thus formed undergoes either

- Transamination - resulting in formation of $\alpha$ ketoglutarate (2-oxoglutarate, which enters TCA cycle) and aspartate (which enters urea cycle);

- Oxidative deamination - in the liver by the enzyme glutamate dehydrogenase resulting in the liberation of free ammonia, which enters urea cycle;

- Decarboxylation - by the enzyme GA decarboxylase resulting in formation of GABA.

\section{Glutamine}

Glutamine is formed in the body from GA and ammonia in an energy requiring reaction catalyzed by glutamine synthase.

$$
\text { Glutamic acid }+\mathrm{NH}_{3} \longrightarrow \text { glutamine synthase } \longrightarrow \text { Glutamine }
$$

Glutamine is strongly concentrated in skeletal muscle making up to $60 \%$ of free amino acid pool in skeletal muscle.

\section{Catabolism}

Glutamine either

- Acts as a nitrogen donor for synthesis for purines and pyrimidines

- Or undergoes deamidation in kidney to produce GA and ammonia. This reaction plays a central role in maintaining acid-base balance in the body and in controlling urinary $\mathrm{pH}^{[2]}$

\section{Natural source}

Glutamate is found in abundance in both 'free' and 'bound' forms in all natural food stuffs - meat, poultry, fish, cheese, milk (including human breast milk), tomatoes, mushrooms, and many other vegetables - peas and broccoli. ${ }^{[1]}$

The bound form of glutamate is linked to other amino acids in proteins present in, muscles, hair, and skin. Glutamate in the human body is considered essential for protein repair, regeneration, and growth. In an average adult, the body content of glutamate is around $1.5-2 \mathrm{~kg}$, mainly in the bound form.

The free form of glutamate (i.e. not linked to protein) in food is known to enhance food flavor and its content is said to increase during natural ripening of fruits. It is not a tenderizer or preservative and cannot make inferior food taste good. It has no distinctive smell. ${ }^{[1]}$

\section{Commercial source for production of glutamic acid}

GA was first produced in Japan in 1908 by hydrolysis of wheat, gluten or Soya bean protein. The fermentation process was invented by Kyowa Hakko Kogyo in 1957, while Ajinomoto Company produced GA synthetically. ${ }^{[11]}$ Large quantities of GA is made by fermenting molasses from sugar beet or sugar cane, a process similar to making wine, beer, sauce, and vinegar. ${ }^{[1]}$

A nonpathogenic species of Coryneform bacterium Corynebacterium glutamicum was originally isolated as an Lglutamate producing bacteria and is now used for industrial fermentative production of various amino acids. ${ }^{[12]}$ Mutations in Its $A$ gene cause a growth defect and induce L-glutamate overproduction by $C$. glutamicum (Fig. 1). Other organisms identified to produce L-glutamate are Brevibacterium, Arithrobacter, and Microbacterium. ${ }^{[1]]}$

\section{Physiology, pharmacology, and therapeutic role of L- GA and glutamine}

\section{Central nervous system}

Glutamate is well established as the principal and classical excitatory neurotransmitter in the central nervous system (CNS). Multiple subtypes of glutamate receptors have been characterized pharmacologically. They are proposed to be both ligand gated and metabotropic receptors. Precise number of subunits and topography of each subunit are not yet established. ${ }^{[10]}$ 
Figure 1. L-Glutamate production pathway reactions within $C$. Glutamicum organism ${ }^{[7],[11]}$

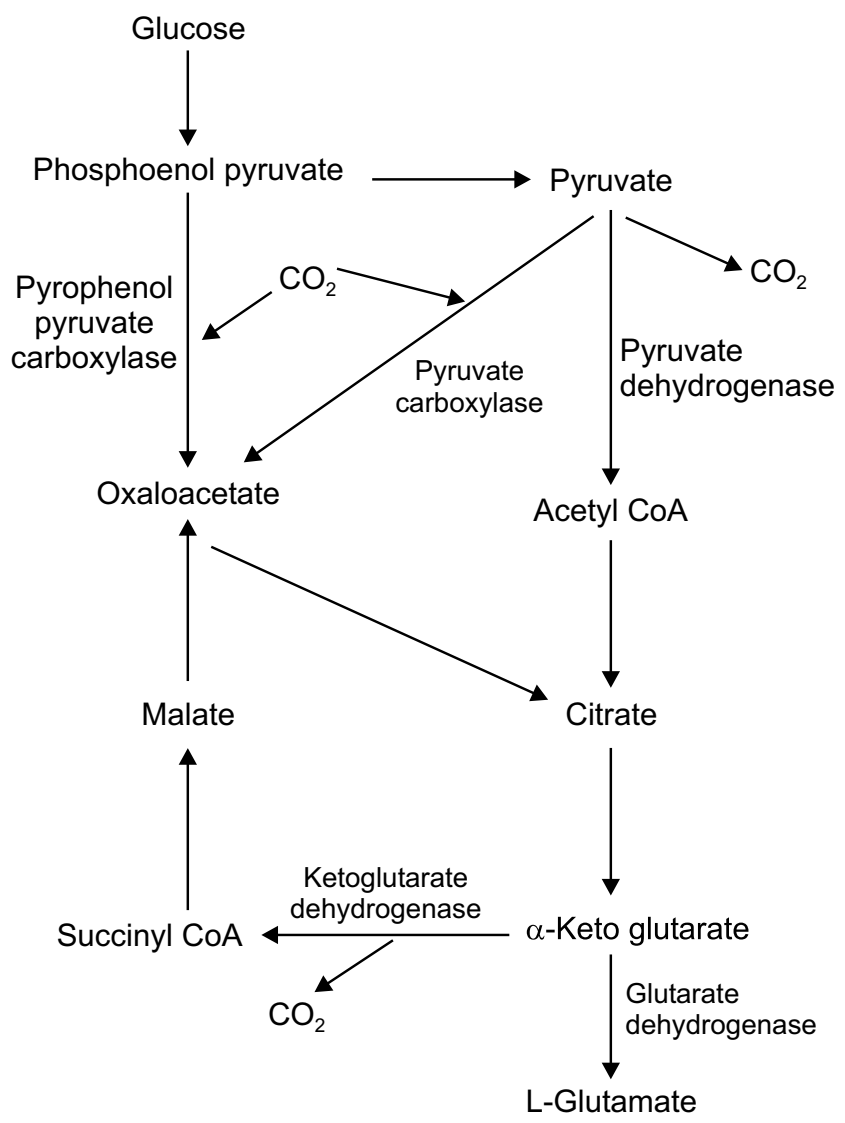

Glutamate through AMPA and kainate receptors mediate fast excitatory synaptic transmission in the CNS and through NMDA receptors, it mediates slow excitatory response, which plays a role in long-term adaptive and pathological changes in the brain involving synaptic plasticity. ${ }^{[13]}$

Elevated levels of extracellular glutamate can induce seizures and excitotoxic neuronal cell death. In glioma and other brain tumors, excessive glutamate release from tumor cells may be responsible for tumor associated necrosis and also possibly to seizures caused due to peritumoral and tumoral brain tissue. ${ }^{[14]}$

A putative abnormality of glutamate metabolism is thought to be implicated in the pathogenesis of amyotropic lateral sclerosis. It is suggested that accumulation of toxic levels of glutamate, an excitatory neurotransmitter in the synapses may cause neuronal death through calcium-dependent pathways in this condition. However, no benefit has been shown with glutamate antagonists, dextromethorphan, and lamotrigine. Several clinical trials with riluzole, which modulates glutamate transmission, have interestingly shown striking improvement in survival among these patients. ${ }^{[15]}$

Imbalance of glutamatergic excitatory control of motor neurons is reported to be responsible for neuronal damage in motor neuron disease; hence antiglutamate agents such as riluzole and gabapentin have been proposed to have protective effects against this neurodegeneration. ${ }^{[16]}$
Table 1

Classification of glutamate receptors ${ }^{[10]}$

\begin{tabular}{|c|c|c|}
\hline Receptor type & Agonist & Antagonist \\
\hline $\begin{array}{l}\text { AMPA ( } \alpha \text {-Amino } 3 \\
\text { hydroxy- } 5 \text { methyl-4- } \\
\text { isoxazole propionic } \\
\text { acid) receptors }\end{array}$ & Quisqualate & NBQX, LY215490 \\
\hline Kainate receptors & Domoic acid & MK801, AP5, LY223053 \\
\hline $\begin{array}{l}\text { N-Methyl-D } \\
\text { aspartate (NMDA) } \\
\text { receptors }\end{array}$ & & $\begin{array}{l}-\alpha-M e-4- \\
\text { Carboxyphenylglycine }\end{array}$ \\
\hline
\end{tabular}

Involvement of multiple neurotransmitter systems is also postulated in relation to Alzheimer's disease, which includes glutamate along with acetylcholine. It is also known that dopamine (DA) interacts with other key systems such as glutamate in Parkinson's disease. This explains the rationale for pallidotomy diminishing the net excitatory input of glutamate on to the dwindling DA neurons and may require further studies. ${ }^{[17]}$

Furthermore, Glutamate is accepted as a precursor for the inhibitory neurotransmitter gamma aminobutyric acid (GABA).

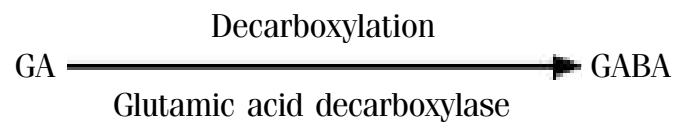

Differential expression of two genes from hippocampal granule cells, encoding different molecular forms of GA decarboxylase (GAD), GAD65 and GAD67 after kainic acidinduced seizures in rat, has been demonstrated. Such progressive and sustained enhancement of expression of both GAD65 and GAD67 messenger mRNA is suggested to augment GABAergic neurotransmission supporting self-protective, anticonvulsive mechanisms in limbic epilepsy. ${ }^{[18]}$

\section{Metabolism}

\section{Ammonia metabolism and nitrogen balance}

Both glutamate and glutamine play an important role in ammonia metabolism. They play a vital role in the disposal of ammonia and thus regulate nitrogen balance in the body. Glutamate plays a central role in transamination reaction in which amino acids funnel their amino group to $\alpha$-ketoglutarate resulting in glutamate formation. Glutamate, therefore, acts as an acceptor of amino group from other amino acids and further undergoes either oxidative deamination in liver or is used as an amino group donor in synthesis of nonessential amino acids.

Glutamine provides a nontoxic storage and transport form of ammonia. The formation of glutamine occurs primarily in the muscle and liver. At all times, there is net output of glutamine from muscle representing the disposal of amino groups from branched chain amino acids. Glutamine formation is a major mechanism for the removal of ammonia in the

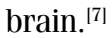

Studies have shown that the glutamine supplementation improves nitrogen balance in patients who underwent major 
surgery or bone marrow transplant. ${ }^{[19],[20]}$ Also a reduced incidence of infection and length of hospital stay have been demonstrated. Further, the lower levels of glutamine in skeletal muscle injuries have been said to indicate its role in the synthesis of muscle protein.

\section{Protein metabolism}

Glutamine plays an important role in protein regeneration, repair, and growth. It is identified as a precursor for purine/ pyrimidine and a substrate for gluconeogenesis in kidney, lymphocytes, and monocytes. The lower levels of glutamine in skeletal muscle injuries have been said to indicate its role in synthesis of muscle protein. This has been the basis for supplementation of stable derivatives of glutamine in total parenteral nutrition (TPN) for patients with injury or infection. Improvement has been attributed to its ability to alter negative nitrogen balance, hepatic protein synthesis, and to reduce protein degradation in addition to a protective role against total peripheral nitrogen-related hepatic dysfunction. ${ }^{[2]}$

Glutamate and glutamine transporters in skeletal muscle and heart appear to play a role in the control of the steadystate concentration of amino acids in the intracellular space probably through osmotic signaling mechanisms to regulate whole body protein metabolism. ${ }^{\text {[21] }}$

The possible role of glutamic acid decarboxylase antibodies (GADA) in type-I diabetes has been suggested in relation to female gender, older age, and the HLA-DR3/DQB*02 haplotype. This conclusion is based on the strong humoral immune response to $\mathrm{GAD}$, which reflects on propensity to general autoimmunity in this condition, rather than excessive beta cell destruction. ${ }^{[22]}$

\section{Reproduction and growth}

Glutamine and glutamate are shown to play an important role in fetal and placental metabolism. ${ }^{\mid 23]}$ Since milk contains good amount of glutamate in free form, it is proposed to be important in postnatal development. ${ }^{21}$ Further, supplementation of glutamine is suggested to offer significant benefits in low birth weight infants including critically ill patients especially in terms of decreased nosocomial infections. $^{[23]}$

Glutathione, a free-radical scavenger synthesized from glutamate in erythrocytes, hepatic tissue, and intestinal mucosa, is found to be depleted in children with low birth weight and kwashiorkor. Therefore, supplementing glutathione and other amino acids have been proposed to be useful in maintaining the integrity of immune system and normal health. $^{[2]}$

\section{Gastrointestinal secretions}

Glutamine is claimed to participate in the maintenance of gut barrier function modulated through the synthesis of nucleotides, glucosamine, and mucus glycoprotein. The GIT is the principal organ of glutamine utilization with $12-13 \%$ of circulating glutamine and 50-60\% of enterally supplied glutamine. Glutamine is considered to act as a major fuel and primary source of energy for enterocytes and to enhance mucosal immunologic function. Its presence is shown to stimulate blood flow to the gut, maintain mucosal integrity, prevent villous atrophy, bacterial translocation, and subsequent pro-inflammatory responses. Gut, the main organ for nitrogen processing in the body has been demonstrated to contribute significantly to the supply of glutamine to peripheral tissues. Impaired gut function is said to be associated with significant fall in glutamine levels, resulting in impaired ability to incorporate amino acids into protein. Clinical trials have shown that glutamine enriched solutions improve nitrogen balance and gut morphology. [2],[19],|23]

There is reported evidence on the reduction in intestinal mucosal permeability and atrophic changes following administration of TPN containing glutamine dipeptide. ${ }^{\mid 24]}$ Glutamine deficiency is shown to be associated with mucosal atrophy and loss of function. In surgical stress, glutamine uptake by the GIT is known to increase. ${ }^{[2]}$

\section{Immunity}

In addition to the role of glutamine in immune function in the gut, it is reported to act as a source of nutrition for cells of immune system released from muscles, adipose tissue and other sources. It is said to help in maintenance of general immunocompetence by supporting synthesis of cellular proteins of the immune system and hepatic acute phase protein response. In sepsis, glutamine uptake, and utilization are known to fall with increase in bacterial translocation. ${ }^{[2]}$

L-Glutamine as precursor of GABA has been stated to have antistress activity and to improve cell-mediated immunity in several studies through nucleogenesis of rapidly proliferating cells. ${ }^{[25]}$ It is claimed that such activity may be useful in the treatment of HIV.

\section{Respiration}

In an animal study, it was demonstrated that GA supplementation reduced and brought heart to body weight ratio to normal which was raised in hypoxia and also GA-treated animals showed higher resistance to fall in rectal temperature than control animals when they were subjected to cold stress. These findings suggest that glutamate in optimal doses may enhance tolerance to hypoxia and cold. ${ }^{[26]}$

\section{Anticancer activity}

The prevalence of cancer-related fatigue in the absence of antineoplastic therapy is said to be $40-75 \%$ and is reported to be due to circulating cytokines such as cathepsin-D and Tumor necrosis factor (TNF) (also called asthenins) are proposed to be responsible for metabolic process underlying muscle mass breakdown observed in cancer patients. It is suggested that nitric oxide could reduce the levels of cathepsin by modulating its synthesis and therefore may benefit cancer patients with fatigue. A double blind placebo-controlled cross over study with oral administration of arginine-glutamate $6 \mathrm{~g}$ /day for 6 weeks was reported to increase the endogenous production of NO in patients with endothelial dysfunction. However, the relation between the asthenins and improvement in fatigue is said to require further studies. ${ }^{[27]}$

In 2000, Oldham et al. have shown superior anticancer activity of paclitaxel an anticancer agent when combined with L-GA in human breast cancer. It's anticancer effect has been attributed to it's ability to produce favorable pharmacokinetic profile and distribution of paclitaxel. The L-GA conjugated with 
paclitaxel is known to form a new anticancer called poly (LGA)-paclitaxel (PG-TXL) with superior antitumor activity, favorable pharmacokinetic properties and/or mechanism of action different from that of Paclitaxel alone. It is suggested that superior activity of PG-TXL may be due to continuous release of paclitaxel. ${ }^{[28]}$

In a double blind placebo-controlled study, administration of GA prevented the vincristine-induced neurotoxicity, a wellknown principal limiting side effect. ${ }^{[29]}$ The loss of tendo-Achilles reflex as an objective parameter of vincristine-induced neurotoxicity was reported to be significantly higher in placebo group as compared to GA group.

Intra-thecal administration of vincristine produced ascending paralysis and death, which was prevented by i.v. GA. Inhibition of disruption of microtubular structures by GA have been proposed to play a role.

\section{Critical illness}

Glutamine has been reported to be of value in the intensive care of critically ill patients. It is shown to improve nitrogen retention, hepatic protein synthesis and reduced protein degradation coupled with increased glutathione and hence protection of enterocytes through antioxidant action and hence is proposed to subserve as an organ-specific amino acid.

It is said that enteral glutamine supplementation preoperatively for 10 days reduces infective complications and length of stay in the hospital. ${ }^{[2]}$

\section{Coagulation}

GA is present in coagulation factors like II, VII, XI, X as Vcarboxyl glutamate residues without which these factors cannot be activated. Hence GA is reported to play an important role in coagulation. ${ }^{[13]}$

\section{Endocrine}

Significant amounts of GA present in prostate gland are said to be implicated in normal function of this gland. In a study involving 45 men, who were given GA in doses of $780 \mathrm{mg}$ for 2 weeks and $390 \mathrm{mg}$ for the next 2.5 months in combination with equal amounts of alanine and glycine, has shown significant improvement in symptoms associated with hyperplasia of prostate. Hence GA is claimed to benefit patients with prostatic hyperplasia. ${ }^{[30]}$

\section{Adverse reactions}

Modern day research completely affirms the safety of sodium salt of GA (MSG) for the general population. ${ }^{[1]}$ Reported evidence suggests the following.

Dose-related adverse reactions

These are not specific for any dose. Symptoms are reported to occur at doses like 1.5 and $12 \mathrm{~g}$ of GA after 15-25 min.

The threshold range for I.V. dose is $25-125 \mathrm{mg}$ for minimum symptoms to occur after 17-20 s. At suprathreshold dose, tightness, pressure over malar, zygomatic, and retrobulbar areas similar to post-anesthetic numbness, burning sensation over chest with or without spreading to the neck, shoulder, forearms, abdomen, and thighs, have been reported in the absence of ECG abnormalities. ${ }^{[31]}$

\section{Sensitivity reactions}

Sensitivity to MSG is shown to be due to the free GA component in food, a consequence of manufacture, following the ingestion of adulterated protein. As per the FDA - the name MSG should be reserved for the ingredient that is 99\% pure combination of GA and sodium. ${ }^{31]}$

\section{Excitotoxicity}

The term is coined to describe the neuronal injury that results from the presence of excess glutamate in the brain, seen in motor neuron disease. Glutamate is required for normal brain function while excess amount leads to neuronal death due to the destructive effect mediated by glutamate receptors, particularly NMDA receptors. ${ }^{[13],[16]}$

The mechanism proposed to be involved includes activated receptor allows influx of $\mathrm{Ca}^{2+}$ leading to activation of destructive process. Also this mechanism is said to be responsible for neuronal death in stroke, ischemia, head injury, and hypoglycemia, during which massive release and impaired reuptake of glutamate in the synapse occurs leading to excessive stimulation of glutamate receptors and subsequent neuronal death.

Seizure

The NMDA receptors are also proposed to be involved in the development of susceptibility to epileptic seizures. Thus modulation of glutamate content is proposed to be beneficial in such conditions. ${ }^{[13]}$

\section{Chinese restaurant syndrome (CRS)}

The literature shows numerous reports of Chinese restaurant syndrome (CRS) in the past. This complex symptom is characterized by burning, tightness, numbness in neck and face, occasionally accompanied by dizziness, headache, nausea, and vomiting, occurring after eating Chinese food. ${ }^{311}$ However, the occurrence of this has been disputed by subsequent studies.

Schaumburg et al. found approximately 30\% population suffered from CRS when fed MSG in ordinary diet due to MSGsensitivity. ${ }^{[32]}$ This needs further evaluation.

\section{Chinese restaurant asthma}

Signs and symptoms of life-threatening asthma were reported in two individuals, 11-14 h after ingestion of MSG in meals. Re-challenge with $2.5 \mathrm{~g}$ of MSG resulted in asthma 11-12 h later. ${ }^{[33 \mid}$ However, strictly controlled studies by university of western Sydney confirmed in 1993 that MSG does not cause asthma attacks. ${ }^{[34]}$

\section{Psychological reactions}

After initial acute reaction to ingestion of MSG followed by long-term psychological effects like depression, drawn facies, motor slowing, doubt-ridden, gloomy fantasies, outbursts of rage, looking strange, and ominous, suggestive of paranoia have been described (called as 'bad dreams'). Bladder and bowel incontinence along with intermittent hyperactivity have been reported. ${ }^{[35],[36]}$

\section{Hyperglutaminemia and hyperammonemia}

Excess consumption of GA leading to brain swelling, astrocyte swelling, and neurological deficit has been reported. ${ }^{[2]}$

No evidence exists till date to suggest that dietary MSG or glutamate contributes to Alzheimer's disease, Huntington's chorea, amyotropic lateral sclerosis, AIDS - dementia complex, 
or any other long term or chronic diseases. ${ }^{[5]}$ Hence, this topic may need further studies.

Miscellaneous

Headache, neurological problems, liver, and kidney dysfunction may occur with high doses. ${ }^{[37]}$

\section{Potential uses}

Taking into account the biochemical, physiological, and pharmacological aspects of L-GA and glutamine, these may be proposed to be useful molecules in several clinical conditions. However, there is no known deficiency of GA and Glutamine encountered in clinical practice.

Food industry

- The MSG is used as a flavor enhancer in savories.

- Use in food is reported to be growing. The MSG is found in most soups, salad dressings, processed meats, frozen entrees, ice creams, frozen yogurt, crackers, bread, in 'low fat' foods to make up for flavor lost when fat is reduced or eliminated. ${ }^{[31]}$

- Maca, a dehydrated, cruciferous root vegetable containing vitamins and essential amino acids including GA, consumed as a dietary substance is said to be beneficial in women to control menopausal symptoms, in osteoporosis, in premenstrual symptoms and used as an energy booster, by doctors in Peru and US. It is said to be beneficial in impotence, chronic fatigue syndrome in men. ${ }^{[38]}$

Gastrointestinal disorders

- In symptomatic treatment of achlorhydria or hypochlorhydria, it is used orally $-0.5-1 \mathrm{~g}$ with meals. The GA hydrochloride is shown to release hydrochloric acid in the stomach.

- Glutamine can be used as a mucosal protective for patients following gastrointestinal surgeries.

- For short bowel syndrome in a dose of 5-10 g dissolved in water 2-3 times a day. ${ }^{[2]}$

- Levoglutamide with alanine by parenteral route is said to maintain postoperative nitrogen balance in patients undergoing major bowel surgery. ${ }^{[19]}$

Cancer

Studies have shown that GA may be beneficial as an adjuvant to anticancer drugs.

- It may act by increasing the efficacy of anticancer drugs. In breast carcinoma paclitaxel conjugate with GA exhibited superior efficacy when compared to paclitaxel alone. ${ }^{[28]}$

- It may act by reducing certain side effects associated with cancer chemotherapy.

- Vincristine induced neurotoxicity was prevented by treatment with $500 \mathrm{mg}$ GA three times a day, orally. ${ }^{[29]}$

- Studies have also shown that GA 6 g/day reduced cancer-related fatigue.

\section{Parenteral nutrition}

In many studies, glutamine has been used as a nutritional agent in parenteral therapy. Since glutamine is unstable, its stable derivatives like glutamine dipeptide or $\alpha$-ketoglutarate were employed and is found to be effective in:

- maintaining cardiac function perioperatively and in promoting glucagon metabolism; ${ }^{[21]}$
- intensive care for the management of critically ill patients;

- following bone marrow transplantation; ${ }^{[20]}$

- following surgery or trauma; ${ }^{[9]}$

- in reducing portal endotoxemia and attenuate hepatobiliary complication, e.g. stone formation; ${ }^{[2]}$

- low birth weight and kwashiorkor. ${ }^{[2]}$

Neurological conditions

In muscular dystrophy, epilepsy, psychiatric disorders, mental retardation, and Parkinsonism the agents, which modulate glutamine transmission are proposed to be useful. ${ }^{[13]}$

\section{Future directions}

Although GA and glutamine have been thoroughly researched as ingredients of food supplies and as nutrients, their evaluation as therapeutic agents appears to be incomplete.

It may thus be concluded that further research is required to explore and examine more closely the potential role of LGA and glutamine as molecules of clinical utility, particularly, in critically ill patients.

\section{References}

1. Msg.org.au [homepage on the internet]. Canberra: Australian Glutamate Information Service [cited 2004 Jul 4]. Available from: http://www.msg.org.au

2. Michele JS, Strain JJ, Benjamin C, Editors. Encyclopedia of Human Nutrition. USA: Academic Press. Harcourt Brace and Company Publisher; 1999.

3. Bellisle F. Glutamate and the UMAMI taste: Sensory, metabolic, nutritional and behavioural considerations. A review of the literature published in the last 10 years. Neurosci Biobehav Rev 1999;23:423-38.

4. Naim M, Ohara I, Kare MR, Levinson M. Interaction of MSG taste with nutrition: Perspective in consumatory behavior and digestion. Physiol Behav 1991;49:1019-24.

5. United States Food and Drug Administration [homepage on the internet]. Rockville MD; [cited 2004 Jul 4]. FDA Backgrounder, Monosodium Glutamate. Available from: http://www.fda.gov/opacom/backgrounders/msg.html

6. Kathleen P. Martindale: The complete drug reference; $32^{\text {nd }}$ edition. UK: Pharmaceutical Press; 1999.

7. Pamela CC, Richard AH, editor. Biochemistry (Lippincott's illustrated reviews). Lippincott Williams and Wilkins. $2^{\text {nd }}$ ed. Philadelphia: A Wolters Kluwer Company; 1994.

8. Msgfacts.net [homepage on the internet]. Chicago: Truth in labelling campaign [updated 2002 Aug 23; cited 2004 Jul 4]. Available from: http://www.msgfacts.net

9. Wernarman, Folke H, Erik V. $\alpha$ - ketogluterate \& Postoperative muscle catabolism. Lancet 1990;335:701-3.

10. Hardman JG, Limbird LE, Gilman AG. Goodman and Gilman's: The Pharmacological Basis of Therapeutics. $10^{\text {th }}$ ed. New York: MCGraw-Hill Medical Publishing Division; 2001.

11. John B, Bjorn K, editors. Basic Biotechnology. New York: Academic press, Harcourt Brace Jovanovich publishers; 1987.

12. Takashi H, Masaaki W, Kazuo N. L-glutamate production by lysozyme-sensitive Corynebacterium glutamicum it's mutant strain. BMC Biotechnol 2001;1:9. http:/ /www.biomedcentral.com/1472-6750/1/9

13. Rang HP, Dale MM, Ritter JM, Moore PK. Pharmacology. $5^{\text {th }}$ ed. Edinburgh. Churchill Livingstone: Elsevier Science Limited; 2003.

14. Ye IC, Rothstein JD, Sontheimer H. Compromised glutamate transport in human glioma cells: Reduction-mislocalisation of sodium-dependent glutamate transporters and enhanced activity of cystine-glutamate exchange. J Neurosci 1999;15:10767-77.

15. Howard RS. Recent advances in neurology. Br Med J 1994;309:392-5.

16. Shaw PJ. Motor neuron disease. Br Med J 1999;318:1118-21.

17. Susan G. Brain drugs of the future. Br Med J 1998;317:1698-701.

18. Schwarzer C, Sperk G. Hippocampal granule cells express glutamic acid decorboxylase 67 after limbic seizures in the rat. Neuroscience 1995;69:705-9.

19. Stehle P, Zander J, Mertes N, Albers S, Puchstein C, Lawin P, et al. Effect of 
parenteral glutamine peptide supplements on muscle glutamine loss and nitrogen balance after major surgery. Lancet 1989;1:231-3.

20. Ziegler TR, Young LS, Benfell K, Scheltinga M, Hortos K, Bye R, et al. Clinical and metabolic efficacy of glutamine-supplemented parenteral nutrition after bone marrow transplantation. A randomized, double-blind, controlled study. Ann Intern Med 1992;116:821-8.

21. Rennie MJ, Ahmed A, Khogali SE, Low SY, Hundal HS, Taylor PM. Glutamine metabolism and transport in skeletal muscle and heart and their clinical relevance. J Nutr 1996;126 (4 Suppl):1142-9.

22. Emad S. Role of antibodies to glutamic acid decorboxylase in type 1 diabetes. Relation to other autoantibodies, HLA risk markers and clinical characteristics. [Academic Dissertation]. Oulu Finland: University of Oulu; 2000.

23. Neu J. Glutamine in the fetus and critically ill low birth weight neonate; Metabolism and mechanism of action. J Nutr 2001;131( 9 Suppl):2585-89.

24. Van der Hulst RR, van Kreel BK, von Meyenfeldt MF, Brummer RJ, Arends JW, Deutz NE, et al. Glutamine and the preservation of gut integrity. Lancet 1993;341:1363-5.

25. Renato $\mathrm{C}$. Glutamine as the key aminoacid in promoting cell-mediated immunity: 20 years of clinical experience. (www.stress-cocchi.org/ Drugs5.htm) (8/28/ 2003).

26. Kumar D, Bansal A, Singh MV, Prasad D, Ilavazhagan G. Improved high altitude hypoxia tolerance and amelioration of anorexia and hypophagia in rats on oral glutamate supplementation. Aviat Space Environ Med 1999;70:475-9.

27. Curt GA, editor. Fatigue in cancer. Massimo M. Under - Rapid electronic response : Dietary Arginine - Glutamate supplementation on plasma levels of Cathepsin: A rationale for a therapeut. Br Med J 2001;322:1560.
28. Oldham EA, Lic KS, Wallace S, Huang P. Comparison of action of paclitaxel and poly (L-glutamic acid) -paclitaxel conjugate in human breast cancer cells. Indian J Oncol 2000;16:125-32.

29. Jackson DV, Wells HB, Atkins JN, Zekan PJ, White DR, Richards F 2nd, et al. Amelioration of vincristine neurotoxicity by glutamic acid. Am J Med 1988;84:1016-22.

30. Amazing herbs [homepage on the internet]. Stone Mountain: [cited 2004 Jul 4]. Natural health library. Available from: http://www.amazingherbs.com/ glutamicacid.html

31. Truth in labeling [homepage on the internet]. Chicago; If MSG isn't harmaful, why is it hidden? [updated 2005 Mar 21; cited 2004 Jul 7]. Available from: http://www. truthinlabeling.org.

32. Schaumburg HH, Byck R, Gerstl R, Mashman JH. Monosodium L-glutamate: Its pharmacology and role in the Chinese restaurant syndrome. Science 1969;163:826-8.

33. Cochran JW, Cochran AH. Monosodium glutamate: The Chinese restaurant syndrome revisited. JAMA 1984;252:899.

34. Allen DH, Baker GJ. Chinese restaurant asthma. N Engl J Med 198;305:1154-5.

35. Tarasoff L, Kelly MF. Monosodium L-glutamate: A double- blind study and review. Food Chem Toxicol 1993;31:1019-35.

36. Colman AD. Possible psychiatric reactions to monosodium glutamate. N Engl J Med 1978;299:902.

37. Any vitamins.com [homepage on the internet]. Gauteng; [cited 2004 Jul 4]. Available from: http://www.anyvitamins.com.

38. Maca roots.com [homepage on the internet]. Murphy; [cited 2004 Jul 4]. Available from: http://www. macaroot.com/science/nutrition.html.

\title{
HEALTHCOM 2005
}

\section{MOBILISING HUMAN RESOURCES \& TECHNOLOGY} FOR ADVANCING THE QUALITY OF LIFE

\author{
16 - 17 August \\ Kuala Lumpur, Malaysia
}

For further information please contact:

\author{
Asian Institute for Development Communication \\ Level 1 Block B \\ Kompleks Pejabat Damansara \\ Jalan Dungun, Damansara Heights \\ 50490 Kuala Lumpur \\ Malaysia \\ T:(603) 2093 8211, (603) 2093 3558, (603) 20932475 \\ F: (603)2093 8567 \\ www.aidcom@streamyx.com \\ www.aidcom.com
}

